\title{
Construção da prática profissional e das mediações documentais do professor documentalista francês
}

Vincent Liquete

\begin{abstract}
Enseignant-chercheur en SIC-Université de Bordeaux-France / EA 487 Cognition et facteurs humains (《Cognição e fatores humanos »). (Ecole Nationale Supérieure de Cognitique)
\end{abstract}

Nos últimos vinte anos, os professores-documentalistas do sistema educacional francês, para a sua profissionalização, cursam dois anos direcionados principalmente às ciências da informação e da comunicação (CIC). Durante o desenvolvimento desta comunicação tentaremos em um primeiro momento mostrar como se constrói progressivamente esta cultura profissional e científica da informação e, em um segundo momento, observar as formas de mediação desenvolvidas no contexto desses atores desde as suas primeiras atividades profissionais em estabelecimentos de ensino. Quatro tipos de mediação parecem predominar entre os professores-documentalistas observados.

Palavras-chave: Cultura informacional; profissionalização; professor documentalista; mediação documental; apropriação dos saberes.

\section{Construction de l'agir professionnel et des médiations documentaires de l'enseignant-documentaliste français}

Depuis ces vingt dernières années, les enseignantsdocumentalistes du système éducatif français suivent au cours de leur professionnalisation, deux années d'enseignement centrées notamment sur les sciences de l'information et de la communication (SIC). Au cours de cette communication, nous tenterons dans un premier temps de montrer comment se construit progressivement cette culture professionnelle et scientifique de l'information pour dans un second temps observer les 
formes de médiation développées sur le terrain par les acteurs, lors de leurs premières activités professionnelles en établissement scolaire. Quatre types de médiation semblent prédominer chez les enseignantsdocumentalistes observés.

Mots-clés : culture informationnelle; professionnalisation enseignant-documentaliste; médiation documentaire; appropriation des savoirs.

\section{Introdução}

Reduzido durante muito tempo à práticas profissionais bastante diversas, os professores documentalistas em atividade nos estabelecimentos de ensino públicos (EPLE, do tipo colégio ou liceu) tentam organizar-se de forma progressiva, delimitando um conjunto de práticas profissionais, que aos seus olhos, constituem um núcleo profissional. A organização daí resultante compreende sobretudo as formações iniciais e contínuas para estudantes e neo-profissionais, bem como um conjunto de reflexões sobre as práticas profissionais desenvolvidas pelos atores. Este domínio de atividade organiza-se, desde os anos 80, em torno de uma prática unificada de profissionalização (concursos nacionais de recrutamento, estruturação de cursos de preparação universitária, ...) e pela implementação de programas de pesquisa científica (Delamotte, 2008) ${ }^{1}$. Uma das principais questões que nos é colocada, consiste na compreensão dos elementos epistemológicos, sobre os quais se constrói esta profissionalização inicial e os saberes nas ciências da informação e documentação, e depois, na observação das formas de mediação documentais destinadas ao usuário, realmente mobilizadas pelos atores, em um momento anterior.

\section{Contexto, elementos teóricos e metodológicos da pesquisa}

\subsection{Contexto da documentação escolar no sistema educacional francês}

A documentação escolar francesa estruturou-se principalmente em torno de duas entidades. Por um lado, o espaço documentário CDI (centro de documentação e de informação), que tem como função o fornecimento dos programas escolares e disciplinares, dos documentos e dos meios de acesso à informação e à cultura em ligação com os programas

\footnotetext{
1 - Comunicação oral em mesa redonda intitulada «Quelles perspectives pour le métier de professeur documentaliste ? » de Eric Delamotte no último congresso da Fédération des Documentalistes et Bibliothécaires de I'Education Nationale (FADBEN), em Lyon em 30 de março de 2008. A ser publicado em 2009.
} 
disciplinares ${ }^{2}$. Por outro, o professor documentalista, enquanto detentor de um Certificado de Habilitação para a prática Professoral e do Ensino de Segundo grau (CAPES) no domínio das Ciências e das Técnicas documentais ${ }^{3}$, certificado nacional destinado a instaurar a profissão e as respectivas práticas profissionais. A criação deste CAPES levou à criação de formações, à escala regional, destinadas aos estudantes, detentores de pelo menos uma licenciatura. Uma reforma nacional prevê, a longo prazo, um recrutamento a nível do mestrado profissional. Deste modo, as universidades que oferecem o curso de Ciências da Informação e da Comunicação (CIC) se encarregaram das preparações para os concursos. A duração média da formação do primeiro ano é de cerca de 450 horas (Liquète, 2006). Depois, num segundo ano de continuação da formação profissional, o estudante torna-se professor estagiário, alternando entre o tempo de estágio EPLE e o tempo de formação, dedicado às questões pedagógicas, documentais e de mediações para os usuários, em cerca de $60 \mathrm{~h}$ anuais ${ }^{4}$. A tudo isto deve-se ainda acrescentar a obrigatoriedade de realização de um relatório profissional, visando à validação de conhecimentos adquiridos, como o certificado de informática e de internet C2i (nível 2). A formação inicial de professores documentalistas tem por objectivo articular nestes dois anos de ensino universitário, os percursos precedentes e as diversas experiências universitárias dos estudantes, com a aprendizagem das $\mathrm{CIC}$, mais precisamente com as questões técnicas e de tratamento informacional e documental, e à problematização ligada à aprendizagem, às questões de educação e de mediação profissional.

\subsection{Questionamento e problematização}

O nosso trabalho tenta caracterizar os principais objetos de estudo informacionais e documentais, escolhidos pelos professores estagiários, ao longo da formação profissional, para poder identificar, depois, as formas de mediação que eles aplicam no campo, através do contato com os estudantes e com os professores da disciplina. Mas, em quais objetos científicos se baseia, essencialmente, a construção de saberes profissionais e que ações de mediação os envolvem prioritariamente?

Em um primeiro momento, tentaremos apresentar as principais formas de apropriação do conhecimento informacional e documental em discussão e depois faremos a caracterização dos quatro tipos de mediação postos em prática, nas primeiras experiências enquanto praticantes.

2 - O CDI do sistema educacional francês cobre as missões documentárias em maior variedade e bem mais que a maior parte das bibliotecas escolares dos outros sistemas educacionais europeus, sobretudo em forte integração com a information litteracy.

3 - Capes propôs por via externa (aberta a todos os estudantes, possuindo uma licenciatura universitária), ou por via interna (para todas as pessoas que podem atestar uma atividade assalariada, dentro da educação nacional, ou em qualquer outra administração, durante três anos escolares). Criação por decreto, publicado no Jornal Oficial da Educação Nacional, a 8 de Julho de 1989.

4 - A partir de 2010, este modo de organização dos estudos será modificado com a conversão em mestrado profissional/mestrado no campo da formação educacional. 


\subsection{Quadro teórico escolhido}

O nosso construto de investigação opta por uma abordagem construtivista, permitindo-nos, assim, compreender as formas de construção de conhecimento aplicadas pelos atores (Andreewsky, Delorme, 2006) e considerar a progressão das práticas informacionais e documentais, como resultado dinâmico de uma busca de equilíbrio e de satisfação das necessidades de informação, entre o sujeito observado e o respectivo ambiente informacional (técnico, humano e metodológico). Cada situação informacional e documental consiste em um confronto entre o conhecimento que o ator possui a princípio e as supostas ou reais potencialidades do seu ambiente informacional. O registo sócioconstrutivista parecia, então, ser adequado ao quadro teórico, para integração do parâmetro da construção entre pares, pelo confronto dos pontos de vista e das representações relacionadas com o ambiente e com a necessidade ou tipo de informação pesquisado. Esta escolha construtivista nos permite relatar as ações e representações dos atores, que estão se formando no domínio de uma cultura científica e técnica, e caracterizar, por fim, as intenções deles, nas ações de mediação realizadas nas primeiras situações profissionais, perante os usuários do CDI.

\subsection{Construto de investigação e métodos utilizados}

Colocamos em prática uma metodologia triangular longitudinal, com a finalidade de apreender o processo de construção, associando três modos de coleta de dados, os quais se complementam entre si e cujo material recolhido será analisado como um conjunto de tendências e comparado à restante informação recolhida. Iniciado no final do ano 2006, este protocolo nos permite atingir três tipos de registos da ação profissional, nomeadamente o do dizer, o do fazer e o sistema de representações dos professores documentalistas. Este estudo faz parte de uma pesquisa nacional Erté. Aqui são colocados, em paralelo, os três modos de coleta de dados que se seguem:

- sessões semi-diretas, realizadas em intervalos de tempo regulares ${ }^{6}$, a partir de uma amostra de 25 pessoas, que foi reduzida para 10 pessoas, assim que saíram, durante o Verão de 2007, os resultados do concurso nacional francês.

- seleções seguidas da análise dos conteúdos produzidos e depois armazenados em arquivos virtuais, em duas formações universitárias observadas (Universidades de Bordeaux e de Pau). Há 18 meses procedemos à análise do corpus dos trabalhos realizados pelos estudantes, tanto sob o ponto de vista da forma como do conteúdo. O

\footnotetext{
5 - A equipe de pesquisa tecnológica em educação (ERTE) intitulada "Culture informationnelle et curriculum documentaire", sob a direção de Annette Béguin, da Universidade de Lille 3 laboratório Gériico: A presentação da Erté pode ser encontrada no site http://geriico.recherche.univ-lille3.fr/erte_information/

6 - A cada seis meses.
} 
nosso intuito é tentar perceber quais objetos de estudo e as principais temáticas escolhidas por estes atores e caracterizar as produções realizadas em colaboração com outros estudantes. É importante salientar que tivemos permissão ao acesso a documentos que circulam entre os estudantes e que foram produzidos por eles próprios, sem a intervenção ou acompanhamento dos docentes;

- por fim, um conjunto de observações realizadas in situ, a partir de dois tipos de usuários, quer no quadro dos projetos envolvendo os docentes da disciplina, quer em situação pedagógica envolvendo os alunos, principalmente durante as sessões planejadas, incidindo sobre as noções da área da informação e documentação. Tais observações centram-se nas condições de transferência e nos elementos de definição e/ou ilustrações escolhidas pelos professores documentalistas, para iniciar e formar no domínio em questão.

\section{A construção dos conhecimentos informacionais e documentais realizada pelos jovens professores documentalistas}

Quais são os elementos mais marcantes observados no desenvolvimento da construção de conhecimentos dos professores documentalistas iniciantes?

\subsection{Uma forte centralidade dos objetos técnicos e específicos}

A formação de estudantes e professores estagiários no domínio dos conceitos e, depois, das situações ligadas às noções da informação e da documentação permanece rara e pontual. Abordadas sobretudo no momento de formação e no trabalho dos estagiários, estas últimas situações encontram-se ligadas à ferramentas e à técnicas de tratamento e de acesso à informação, mais precisamente à informática documental e às ferramentas de pesquisa na Internet. O limite dessas formações é que elas, antes de mais nada, exigem um domínio técnico destes instrumentos e não consideram para análise, os objetos sociais e técnicos, formatando e orientando a procura de informação dos usuários e suas necessidades e desafios sociais, econômicos, ... O objetivo destas ações é, afinal, permitir que os estudantes aumentem a sua autonomia durante as pesquisas, mesmo que o nível de questionamento sobre os modos de pesquisa, de classificação e de apresentação, sejam abordados de forma muito sucinta. As técnicas propostas pelos jovens praticantes são consideradas, até hoje, instrumentos de performace, não constituindo nenhuma análise nem crítica.

Os conhecimentos do domínio da informação e da documentação, abordados em aula, relacionam-se aos princípios positivistas, de definição, compreensão e de interpretação, mas não traduzem o carácter dinâmico, instável e complexo da informação e do documento. 
Finalmente, um grande número de estudantes entrevistados afirma que a maior parte das noções assimiladas durante o primeiro ano de formação, de carácter informacional e científico, revelam apenas estar ligadas a um contexto político e institucional prescritivo, procurando estimular, no campo, por meio da documentação escolar, novas iniciativas e modalidades de gestão da informação, no interior dos estabelecimentos escolares. A "política documental", a "prospecção documental" e o "projeto documental" são bons exemplos desse caso. Alguns estudantes chegam mesmo a declarar que sentem que uma parte da sua formação, na área da documentação, contribui para uma melhor formação em termos políticos e de gestão, do que em termos científicos e informacionais.

\subsection{Objetos e posicionamentos epistemológicos ausentes}

Alguns trabalhos provaram, nestes últimos meses, que as teorias da informação/comunicação eram abordadas muito superficialmente, durante os anos de formação profissional dos professores documentalistas (Liquète, 2006), ou eram até mesmo desconhecidas, pelos profissionais em atividade (Blanquet, 2003). A espinha dorsal das formações iniciais universitárias é constituída pelos três eixos que se seguem: o da informação transformada (seleção, descrição, análise e até reescrita), o da informação recuperada (pesquisa e subsequente recuperação, tipologia dos recursos) e o da informação produzida (abordagens sistêmicas, atores, canais de difusão). As primeiras formações profissionais propõem conteúdos, de finalidade essencialmente técnica, em detrimento de objetos de incentivo à compreensão dos fenômenos complexos humanos ligados à informação, tais como a identificação e a análise dos usos, os desafios da produção veiculada pelas indústrias culturais ou escolares, ou a análise das representações de profissionais da documentação. Além disso, detectamos objetos epistemológicos ainda surpreendentemente ausentes no ensino ministrado. São eles:

- a caracterização das práticas de informação e de documentação dos diferentes públicos, especialmente o dos jovens, incluindo os de natureza não-formal, tais como as práticas sociais comuns, mas eficazes na satisfação que proporcionam no dia-a-dia e estruturantes em termos de sociabilidade (Tough, 1979);

- a análise reforçada das tecnologias (Jeanneret, 2000) e das mídias, como meios de formatar e estruturar o pensamento;

- o questionamento do indivíduo "pesquisador de informação": numerosos participantes formulam o princípio de que o indivíduo (aluno, professor, cidadão,...) é intimamente um pesquisador de informação, sujeito à necessidades constantes de informação (Le Coadic, 1998).

Para concluir este tópico, podemos sublinhar que a ausência de uma atitude analítica e reflexiva pode vir a limitar as formas e a diversidade das mediações envolvidas no campo, pelos professores documentalistas.

\subsection{Práticas colaborativas escondidas}


Tal como salientamos anteriormente, numa perspectiva sócioconstrutivista, a análise da construção e da transferência de conhecimentos dos indivíduos, obriga a um questionamento das suas interações. Consideramos, então, as interações entre os documentalistas estagiários, fora de toda e qualquer prescrição dos seus formadores. A maioria $(79 \%)$ indica que o trabalho realizado, no interior do grupo a que pertence, constitui a melhor maneira de exercer a profissão de documentalista.

Durante as nossas entrevistas, uma estagiária relembra: « dentro do grupo de trabalho a que pertenço, aprendo facilmente a prática da profissão de doc (CIC)». Após a análise e o recorte dos dados produzidos, são mobilizadas três tipos de competência documental, de forma prioritária, a saber:

Monitorar e reescrever informações editoriais difundidas através de diversos canais (imprensa profissional, sites profissionais ou associativos, ...). Este trabalho envolve, progressivamente, atividades de monitoramento e prospecção documental.

Courrier hetrinational $\mathrm{n}^{\mathrm{a}} 906-\mathrm{du} 13$ au 19 mars 2006

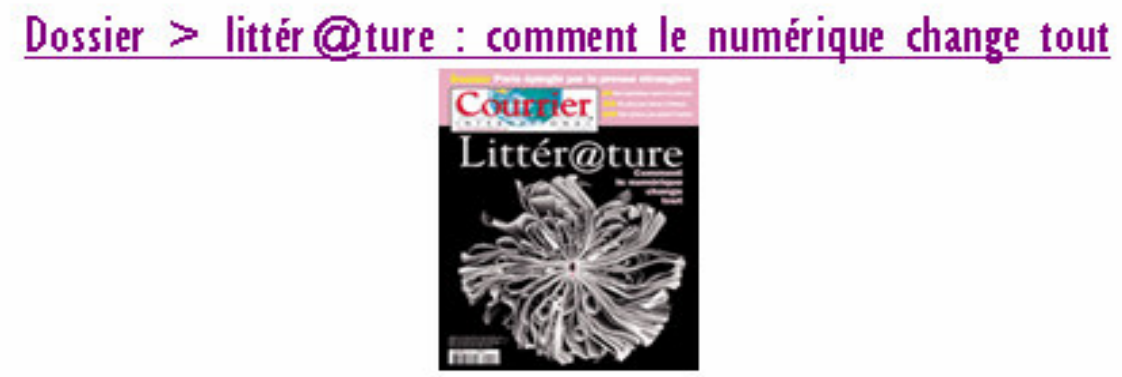

Editorial (trabele lawais

tprès le anéma, la photographie et la musique, est-ce au tour du livre d'entrer dans la révolution numérique ?

Oblige à repenser les pratiques d'éciture, d'édition et de lecture.

La tablette liodfe, lanóe en novembre 2000 , s'est vendue à près de 10000 exemplaire et est en rupture de stodk

La fin du livre n'est pas pour demain ihidoass meè

I y a toujours eu des casandre pour prédire la fin du livre. Mais le lire se débat comme un beaus diable. Les rentes continuent de progresser.

Les rentes de dictionnaires et de guides pratiques ont diuté de $40 \% /$ depuis 2003 . Dans le secteur de l'édition scientifique et tednique, la révolution tedinologique est d'ores et déjà en mardie.

Estrato de um relatório de monitoração, realizado por um grupo de estudantes, em 2008.

- Otimizar os sites ligados às questões educacionais e às aprendizagens da disciplina, sejam elas profissionais, de associações ou institucionais; estas especializações incidem simultaneamente sobre a análise dos conteúdos e sobre a sua estética, ergonomia e navegabilidade.

Ligar, organizar e hierarquizar os conteúdos absorvidos durante a formação, através de leituras pessoais no domínio das CIC e, principalmente, das ciências da educação ou, ainda, nas atividades de monitoramento. Por iniciativa dos próprios estagiários, são propostas, diversas atividades, das quais são exemplo os modelos mentais (cf. esquema que se segue) ou índices de noções. 


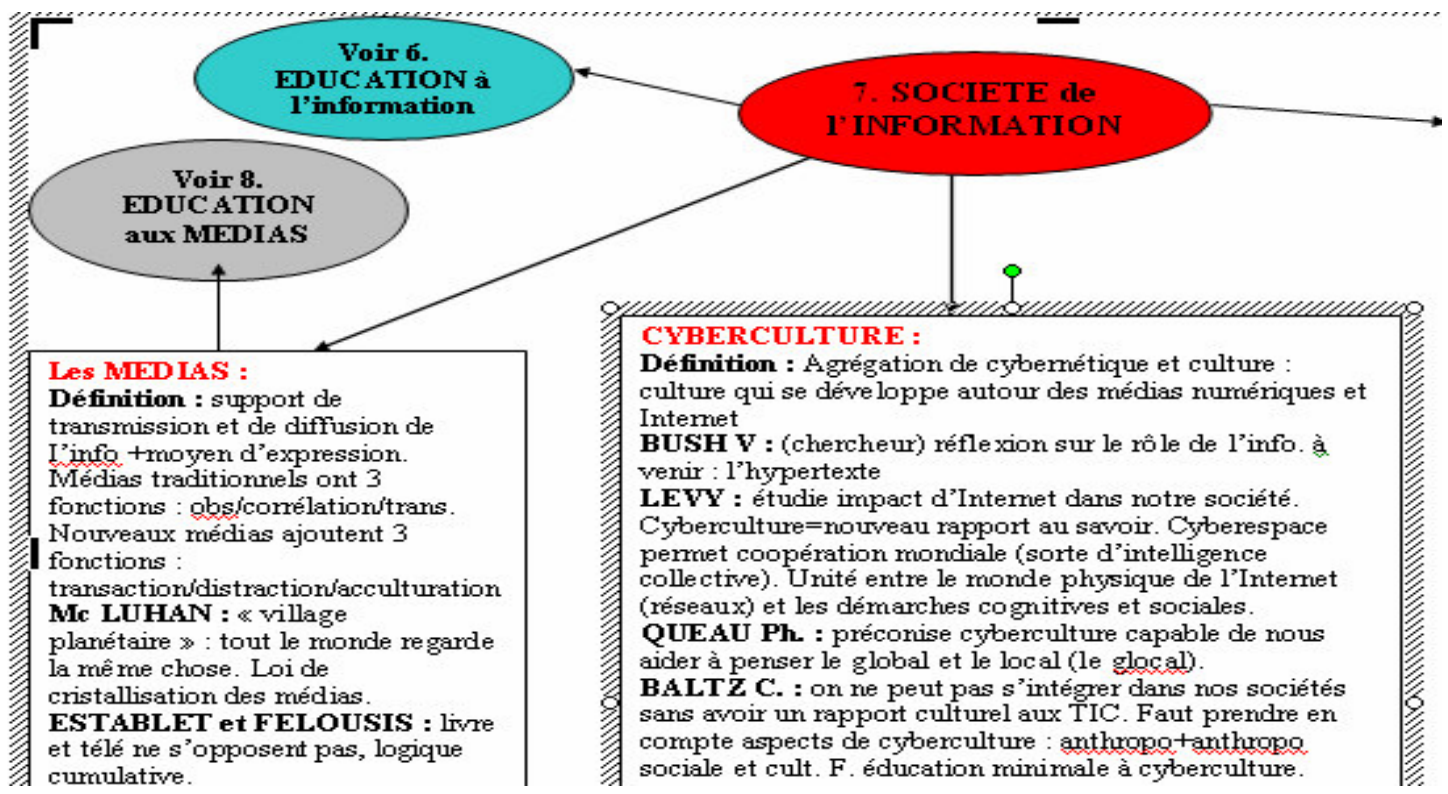

Extracto de um modelo mental analítico (sob a forma de janela digital) realizada em 2008, por um grupo de estudantes.

O essencial das suas atividades de construção de conhecimento sustenta-se, assim, sobre estas três formas de trabalho, que revelam também os modos de participação, organização e compartilhamento de informação bem elaborados, atingindo portanto a aquisição progressiva de uma cultura documental profissional compartilhada.

\section{As formas de mediação envolvidas engajadas}

No momento em que os setores da educação encontram-se também expostos a ofertas diretas de informação dos editores difusores e utilizadores, e tudo isto sob ofertas extremamente variadas ${ }^{7}$, podemos nos questionar sobre os posicionamentos tomados pelos novos documentalistas, como eco a sua formação e aos seus percursos infodocumentais. Manterão eles, na verdade, atividades de mediação e sob que formas elas se organizam? Quatro tipos de mediação predominam nos EPLE observados:

\subsection{Mediação informacional pró-ativa}

Se aceitarmos a ideia de considerar os passos da antecipação da oferta informacional como um «processo contínuo e dinâmico, encarregado da oferta periódica e personalizada de dados (...), tratados de acordo com a própria intenção do destinatário, recorrendo à opinião de um perito, em relação com o assunto ou com a natureza da informação coletada » (Collectif ADBS, SCIP, SYNAPI, 1995), constatamos que as atividades empreendidas pelos professores documentalistas baseiam-se

\footnotetext{
${ }^{7}$ Por exemplo, o pacote de serviços como o Canal Numérique des Savoirs (CNS, www.cns-edu.net ), explicações extracurriculares em linha e trabalho pessoal do aluno, os editores institucionais (rede Scéren-

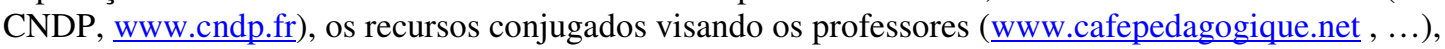


nestas intenções, mas que acabam por ser produções e atividades bem mais modestas. Ela surge a partir da intenção de agrupar um conjunto de informações de carácter profissional, ligadas à atualidade da profissão, de um público de docentes que possui dificuldades de acesso a estas informações, por falta de competências, falta de tempo ou de estratégias de organização. Nós qualificamos esta atividade como "mediação próativa", pelo fato dela sustentar-se em dois princípios: o de apenas filtrar informação de carácter profissional, e sobre uma atuação ativa, por parte do documentalista que a seleciona, a organiza e a divulga ao públicoalvo ${ }^{8}$. A antecipação da oferta informacional encontra-se organizada em torno de três registros de intenção, que são: um interesse central partilhado entre pares, um projeto pedagógico a realizar ou a pesquisa de um " boa experiência" relacionada com a disciplina escolar de pertencimento (Delamotte, 2007). Os jovens professores documentalistas entrevistados declaram colocar em prática, este tipo de mediação, muito rapidamente, no campo de atuação, transferindo os métodos e os saberes práticos, adquiridos com o trabalho pessoal e colaborativo, empreendido durante a formação9. Além do mais, estão disponíveis na Web, ofertas de mediação pró-activa mais elaboradas, resultantes de ações empreendidas por jovens profissionais ${ }^{10}$

\subsection{A mediação cultural}

A maior parte dos documentalistas acompanhados investe no setor cultural, com a finalidade de por em prática actividades de mediação. Nós nos reteremos na proposta de definição do portal do Ministério da Cultura Francês, relembrando que a mediação cultural "consiste em suscitar o desejo e proporcionar os meios de acesso às práticas culturais e artísticas, em todos os domínios. (...) ela tem por objetivo facilitar o acesso à arte através da execução de ações específicas, destinadas a públicos definidos". As atividades empreendidas procuram, por sua vez, permitir aos estudantes principalmente, que descubram os recursos culturais colocados a sua disposição, mas visam também o processo interpretativo, permitindo-Ihes dar sentido as suas novas descobertas. Não se trata apenas de ficar no nível da apresentação e da selecção de recursos; na verdade, os documentalistas observados tentam colocar os criadores, órgãos de referência, museus, em contato com as necessidades e projetos dos públicos do CDI. Esta mediação cultural repousa na difusão de informações, nos encontros de artistas ou de representantes institucionais $e$ até mesmo nas ações de animação e de produção em diversos segmentos das artes. Por mais ativa e militante que seja, esta mediação

8 -Entretanto, não podemos dizer que essas informações disseminadas sejam personalisadas ou respeitem uma periodicidade rigorosa.

9 - Um complemento para a mediação pró-ativa é reorientar a política de aquisição do acervo documental do CDI.

10 - Extrato de um portal de antecipação de informação da imprensa sobre netvides realizado por um jovem professor documentalista ( na IUFM, Aquitânia, em 2006). Onkline (http://www.netvibes.com/kiosquelycee Home) 
do professor documentalista não é neutra, nem ocupa uma "tierce position" (Six, 1990); mas situa-se diretamente do lado dos professores, que participam de certa maneira para reforçar a cultura artística acadêmica, ligada aos programas oficiais de ensino. Todavia, as atividades de compreensão e de produção envolvidas não permitem analisar satisfatoriamente os códigos e as formas de interpretação e apropriação das obras.

\subsection{A mediação através de « noções informacionais 》}

Nos anos 90, os professores documentalistas introduziram na formação dos alunos, o ensino de técnicas e de instrumentos documentais, acessíveis principalmente a partir dos CDI.

Essas iniciações foram objeto da criação de situações e exercícios pedagógicos, que constituíram progressivamente uma pedagogia documental (Béguin, 1996). Estes últimos anos ficaram marcados por uma importante reorientação, que tinha por finalidade, ultrapassar o único registro da pedagogia documental, para impulsionar uma "didática de informação", cujos desafios seriam simultaneamente técnicos (domínio das técnicas de tratamento e acesso à informação), analíticos (conhecimento da informação, a sua história e os próprios desafios) e comportamentais (adoção de posturas reflexivas, comparativas e críticas, do ponto de vista informacional dos conteúdos informacionais que o circundam). Esta prática baseia-se, de igual modo, em experiências provenientes do ensino superior ou de áreas específicas do ensino, tais como o ensino agrícola (Gradiès, 2006). Como sugere V. Couzinet (2008: 185) a visão ultrapassa o domínio único da informação, já que " trata-se de, em seguida, de fazer os utilizadores dos centros documentais, descobriem noções, questionamentos e saberes fundados em conhecimentos construídos por pesquisadores, que Ihes permitam adquirir uma cultura da informação, da mesma forma que já dispõem de uma cultura histórica ou geográfica, por exemplo".

A partir de então, a palheta de atividades do professor documentalista, ultrapassaria além da simples gestão à oferta de mediação, passando igualmente a propor situações de transmissão de conhecimentos.

Os jovens documentalistas acompanhados desde 2006, tentam realizar atividades em que através da ajuda de exercícios ou situações, propiciem que os alunos apreendam progressivamente noções chave do campo da informação e da documentação. Esta "mediação-transmissão" em torno de noções ou conteúdos informacionais ${ }^{11}$ baseia-se principalmente nas quatro ações que se seguem:

- o objetivo de propor aos alunos uma definição que lhes permita delimitar e apropriar-se de um conteúdo;

- à medida que os conteúdos são abordados, tentar colocá-los em ligação uns com os outros, reagrupando-os de acordo com conceitos

11 - Uma visão de noção info-documental está disponível no «Dictionnaire de notions », [online] em: http://savoirscdi.cndp.fr/culturepro/actualisation/Duplessis/dicoduplessis.htm 
integradores da autoridade. Este tipo de atividade apoia-se em experiências anteriores dos estudantes;

- a proposta de exercícios de aplicação, buscando reforçar a apropriação de conhecimentos da área do conteúdo estudado.

- por fim, a apresentação de ferramentas, de meios ou de documentos que se encontram à disposição dos estudantes (online, a partir do sistema de informação EPLE ou no CDI), que lhes permitam continuar a adquirir conhecimentos, a partir do conteúdo abordado.

Esta forma de mediação, a partir do estudante, possui a vantagem de edificar progressivamente, todo um acompanhamento construtor em torno da informação e que ultrapassa o único modelo único de transmissão de conhecimento. Salientamos, contudo, que não estão sendo considerados os centros de interesse ou de preocupação do estudante e que não procuramo analisar em profundidade nesta pesquisa para depois compreender, os usos e as práticas (muitas vezes intuitivas) autônomas do estudante (Dioni, 2008).

\subsection{A mediação através « redocumentação 》}

Uma nova forma de mediação destinada à informação e aos conhecimentos, distingue-se não mais pelo documento disponibilizado pelo professor documentalista, mas pelo (re) tratamento documental que é trabalhado prioritariamente pelo usuário. Nós entendemos por "redocumentação", a definição dada por Manuel Zackland ${ }^{12}$ de que

"redocumentar é documentar de novo um documento (...) permitindo que o beneficiário reestruture os conteúdos semióticos, de acordo com a sua interpretação, sua forma de uso, com os objectivos que motivam a sua ação e que podem ser o da construção do seu próprio conhecimento ou o de constituição de um conjunto de novos recursos, para a comunidade de interesse a qual ele pertence". 


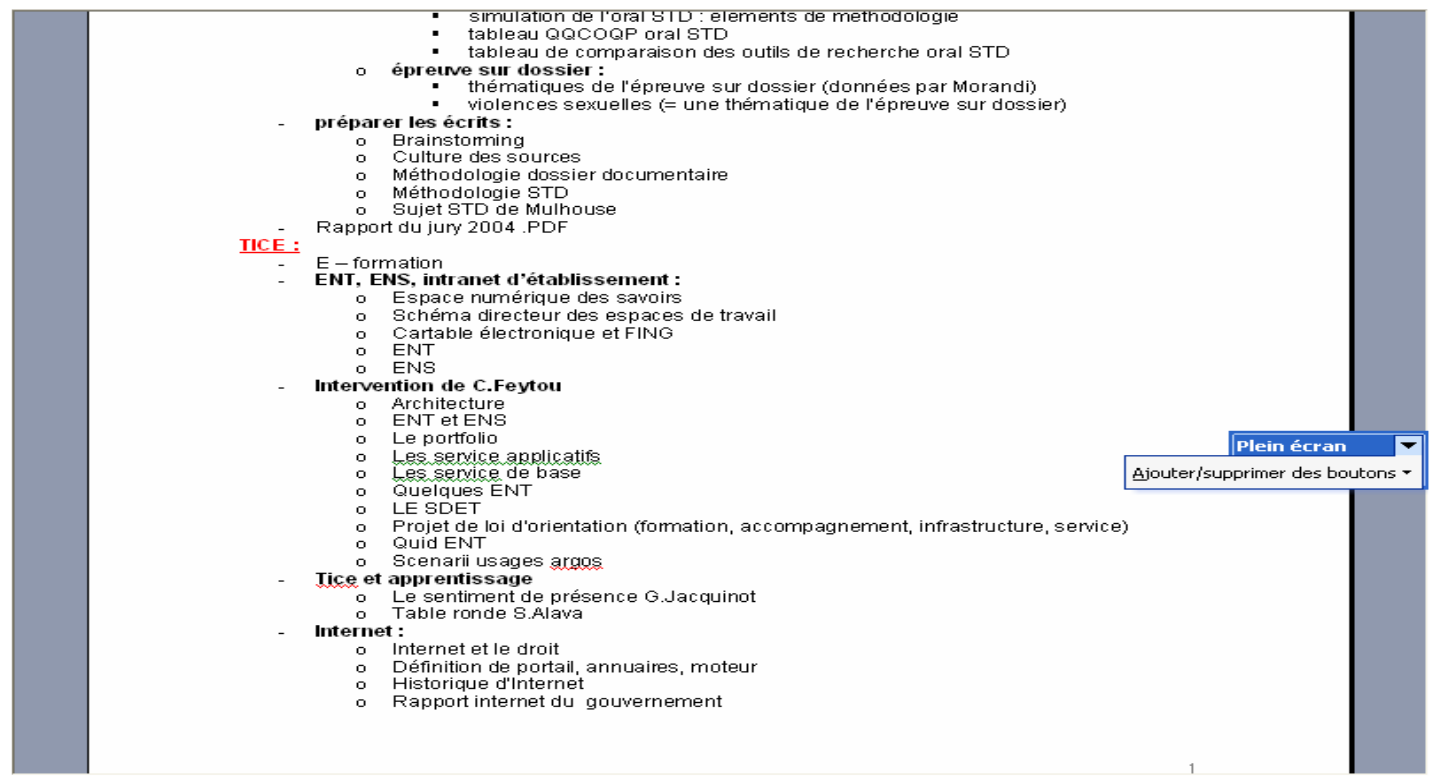

Exemplo de construção arbórea sob a forma de índice alfabético, combinando diversas terminologias, a partir da associação de leituras, documentos, atividades de antecipação informacional e de ensinos de acompanhamento presencial.

Estes métodos de aprendizagem, validados por estudantes $\mathrm{e}$ estagiários, servem de embasamento às primeiras experiências de práticas profissionais em contexto pedagógico, durante 0 ano de professor-estagiário. Uma estudante, Agnès ${ }^{13}$, nos declarou que "este tipo de recurso muito me ajuda, na escolha das minhas aulas de pedagogia documental (...) porque, depois de ter passado no concurso, deixei de dar tanta atenção ao meu trabalho pessoal e, agora, graças a este tipo de recurso, tenho não só uma base comum de trabalho com os colegas, mas também uma indicação geral daquilo que poderei trabalhar com os alunos, nas aulas.".

Esta tendência geral observada, demonstra que os estudantes organizam de maneira metódica as diversas atividades realizadas no primeiro ano, para poderem estruturar e delimitar as suas primeiras formas de prática profissional, que constituem também uma espécie de "memória info-profissional" e de mediação para os futuros usuários. Assim, esses atores passam, de uma cultura de interesses, a uma cultura (profissional) de grupo. A mediação, através da « redocumentação », coloca-se também em grande parte nos procedimentos de "reconfiguração". A "reconfiguração" dos documentos consiste na alteração progressiva da finalidade inicial de certos documentos, mais precisamente dos que são entregues pelos professores das formações; eles são, essencialmente, de tipo metodológico e são elaborados por eles no momento dos cursos ou em trabalhos dirigidos, depois reutilizados e, finalmente, reapropriados pelos estudantes, para as resenhas de leitura, para os exercícios de síntese ou para as apresentações de temas. Tratase, portanto, de um tratamento informacional do tipo "reconsignação",

13 - Respeitando o anonimato, os nomes citados não correspondem à identidade real dos estudantes e professores-estagiários entrevistados. 
considerando-se que as ações e as intenções metodológicas iniciais são contornadas e reapropriadas pelos grupos, com o objectivo de organizar suas próprias estratégias anteriores de trabalho e de produção.

\section{Conclusão}

Como pontuado por M. Gellereau (2006: 41), existe um risco de «cair numa espécie de "mediacionismo", em que tudo se explica(ria) pela mediação». Tal como as atividades culturais indicadas pelo autor, as ações pedagógicas e documentais escolares, não passam exclusivamente pelas atividades de mediação. Entretanto, as que foram observadas estruturamse em torno de um conjunto de estratégias variadas, como a formação, a produção de informação ou animação e o contacto com o ambiente EPLE, que reforçam a apropriação de conhecimentos. Por outro lado, podemos notar que as ações de mediação baseiam-se em um conjunto de experiências anteriormente adquiridas, durante as atividades compartilhadas entre estudantes, em construção conjunta, mais do que naquelas aprendidas nos cursos na universidade. Compreender e analisar as formas das práticas do agir profissional, leva-nos a considerar o conjunto das acções de mediação para o usuário, nas quais estão envolvidos os modos comunitários de organização dos conhecimentos determinados pelos estudantes.

\section{Agradecimentos :}

O nosso muito obrigado, a todos os estudantes e professores estagiários envolvidos nesta pesquisa, por aceitarem ser observados e entrevistados ao longo de todos estes meses.

Assistente à tradução: Simone Miranda (University of CoimbraPortugal)； simone_miranda_fr@yahoo.fr

\section{Referências}

ANDREEWSKY, Evelyne, DELORME, Robert (dir.), 2006. Seconde cybernétique et complexité : rencontres avec Heinz Von Foerster. Paris : L'Harmattan.

BEGUIN, Annette, 1996. Didactique ou pédagogie documentaire. Ecole des lettres-collège, $n^{\circ} 12$ spécial, juin, p. 49-64.

BLANQUET, Marie-France, 2003. Facteurs d'évolution et avenir de la profession d'enseignant documentaliste. 11 p. [En ligne] http://docsdocs.free.fr/spip.php?article63

CACALY, Serge, [et al.], 2004. Dictionnaire de l'information. Paris : Armand Colin, p. 148-149.

COUZINET, Viviane, 2008. De l'usager à l'initié: vers une culture informationnelle partagée In GARDIES, Isabelle [et al.] (dir.). Education à l'information et éducation aux sciences : quelles formes scolaires? : 
rencontres Toulouse Educagro'08, Castanet Tolosan, 26-27 mai 2008. Toulouse : Cépaduès, p. 169-189.

DELAMOTTE, Eric, 2007. Configuration des connaissances à l'ère des $\begin{array}{llllll}\text { communautés numériques. } & 9 & \text { p. } & \text { [En } & \text { ligne] }\end{array}$ http://www2.toulouse.iufm.fr/flam/thot/docs/delamotte.pdf

DIONI, Christine, 2008. Métier d'élève, métier d'enseignant à l'ère numérique. Lyon : INRP.

FONDIN, Hubert, 2001. La science de l'information: posture épistémologique et spécificité disciplinaire. Documentaliste-science de l'information, vol. 38, n²2, p. 112-122.

GARDIES, Cécile, 2006. De la mouvance identitaire à l'ancrage scientifique des professionnels de l'information-documentation dans l'enseignement agricole : thèse de doctorat. Toulouse : Université du Mirail. 373 p.

GELLEREAU, Michèle, 2006. Pratiques culturelles et médiation. In Sciences de l'information et de la communication: objets, savoirs, discipline/ Olivesi, Stéphane. Grenoble : Presses universitaires, p. 27-42.

JEANNERET, Yves, 2000. Y-a-t-il (vraiment) des technologies de l'information ? Lille : Presses universitaires du septentrion.

LE COADIC, Yves-François, 1998. Le besoin d'information : formulation, négociation, diagnostic. Paris : ADBS édition.

LIQUETE, Vincent, 2006. L'incidence des enseignements en SIC sur les pratiques professionnelles de l'enseignant-documentaliste du système éducatif français. In SFSIC. Questionner les pratiques d'information et de communication : agir professionnel et agir social, Bordeaux, 10-12 mai $2006,15^{\text {ème }}$ congrès, p. 387-395.

SIX, Jean-François, 1990. Le temps des médiateurs. Paris : Seuil.

TOUGH, Allen, 1979. The adult's learning project: a fresh approach to theory and practice in adult learning. Toronto : The Ontario institute for studies in education. 